\title{
Catalytic/inhibitory effect of the joint presence of two dyes on its destruction by underwater plasma processes: a tool for optimization parameters of treatment
}

\author{
Anna Khlyustova ${ }^{1}$ (1) Anna Grosheva ${ }^{2} \cdot$ Nikolay Sirotkin $^{1} \cdot$ Daria Panova $^{2}$
}

Received: 1 August 2019 / Accepted: 17 October 2019 / Published online: 24 October 2019

(c) Springer Nature Switzerland AG 2019

\begin{abstract}
The underwater plasma was used for the destruction of two-dye mixture. The Direct Blue azo dye and xanthene dye of Rhodamine $6 \mathrm{G}$ used as the model pollutants. The concentration of one of them is varied for modeling the processes in which one of the classes of the dye exceeded other dyes. The catalytic/inhibitory effect of the joint presence of two dyes is studied. The effect of initial temperature and the role of $\mathrm{H}_{2} \mathrm{O}_{2}$ in the mechanism of destruction were also investigated. It was established that the processes of decay of organic dyes proceed through the stages of the formation of intermediate products, which are catalysts or inhibitors of further degradation processes. An increase in the temperature of the solution has almost no effect on the degree of decolorization of the solution of the dye mixture. It is established that the destruction of Direct Blue 1 occurs under the action of hydrogen peroxide or $\mathrm{HO}_{2}$ radicals, while Rhodamine $6 \mathrm{G}$ is destroyed by interaction with hydroxide radicals. The comparison of energy yield values with published data showed that underwater plasma is an effective method for removing the organic dyes from wastewater.
\end{abstract}

Keywords Underwater plasma · Destruction · Dyes mixture · Rhodamine 6G · Direct Blue 1 - Energy yield

\section{Introduction}

Dyes are complex organic compounds that are used in various industries (textile, chemical, dyeing, electronics, etc.). The disposal of organic compounds from wastewater is one of the main issues of treatment. Recently, there has been significant interest in using advanced oxidative processes to solve this problem [1-5]. Of interest are developments based on the use of nonthermal plasma in contact with a liquid, as reflected in recent papers [6-9].

There are many features of different types of electrical discharges. For example, the gliding arc discharge generates the nonthermal plasma in the gas phase with a high concentration of charged and neutral reactive species. On the other hand, the replacement of the plasma zone in a liquid volume and creating underwater discharge have some advantages in comparison with electrical discharges above liquids. It is a large size of the contact of plasma zone and liquids, convective flows, and cavitation effect.

There are a lot of data in which the destruction of single dyes by electrical discharges treatment is studied [10-39]. One of the most popular objects of research is methylene blue dye, which is one of the thiazine dyes $[10,12,16,18,21]$. A large number of works are devoted to studying the effect of electric discharges on azo dyes due to the widespread use of this class of dyes $[14,15,17$, $19,25-27,30,32,34]$. It should be noted that the growth of studies on the decomposition of anthraquinone dyes because of carcinogenic properties in water [20, 23, 24, $29,31,33,35]$. However, the wastewater of industrial factories contains more than one dye. It is the mixture of the organic and inorganic compounds. At present, there are

Anna Khlyustova, kav@isc-ras.ru| ${ }^{1}$ G. A. Krestov Institute of Solution Chemistry of RAS, Ivanovo, Russia $153045 .{ }^{2}$ Ivanovo State University of Chemistry and Technology, Ivanovo, Russia 153000.

SN Applied Sciences (2019) 1:1465 | https://doi.org/10.1007/s42452-019-1516-y 
some references regarding the application of nonthermal plasmas to a mixture of the organic dyes [39-42]. In [42], it was found that the destruction of two dyes occurs faster in their mixture than in the solutions, which contain only one dye. It was assumed that the dyes in a mixture or its byproducts could be a catalyst in the degradation process.

In this work, the object of research was a plasma treatment of a mixture of dyes Rhodamine $6 \mathrm{G}$ and Direct Blue 1. The choice of dyes was determined by their belonging to different classes of dyes. Rhodamine $6 \mathrm{G}$ is a representative of the class of xanthene dyes, which are used in both the textile and electronics industries. Direct Blue dye 1 is an azo dye. Its choice is explained by the fact that about $70 \%$ of the dyes used in the industry account for this class of dyes. The underwater diaphragm discharge was used as the nonthermal plasma. The main goal of this paper is to investigate the effect of a concentration of azo dye on decolorization efficiency and the rate of the destruction of dye's mixture by underwater plasma treatment. Varying the concentration of azo dye concerning Rhodamine $6 \mathrm{G}$ models processes in which the content of azo dyes exceeds the concentration of other classes of dyes. Based on the early published data, we suggest that the presence of two or more organic dyes may induce the catalytic effect for fast degradation of both dyes. In the present work, we try to prove this assumption. The effect of the initial temperature of a solution was also studied.

\section{Materials and methods}

\subsection{Materials}

Rhodamine 6G (Rh6G) and Direct Blue 1 (DB) (Reachim, Russia) were used without any future purification. The molecular structures are shown in Table $1 . \mathrm{HCl}, \mathrm{KMnO}_{4}, \mathrm{KI}$, $\mathrm{NH}_{4} \mathrm{MoO}_{4}, \mathrm{Na}_{2} \mathrm{~S}_{2} \mathrm{O}_{8}$, and oxalic acid were purchased from Reachim (Russia) and used as received. All chemicals were p.a. grade. All solutions were prepared using deionized water. To make the conductivity of a solution, the $\mathrm{HCl}$ is used (adjusted to $\mathrm{pH}$ 6.5). Conductivity of solution was $60 \mu \mathrm{S} \mathrm{cm}^{-1}$.

Aminoquinone was synthesized in laboratory at Department of Fine Organic Synthesis (Ivanovo State University of Chemistry and Technology).

\subsection{Experimental setup}

Schematic diagram of the experimental setup is shown in Fig. 1. An AC high-voltage transformer $(50 \mathrm{~Hz}, 220 \mathrm{~V} / 1 \mathrm{kV})$ was used to generate the underwater diaphragm discharge. The input power was $75 \mathrm{~W}$. Two graphite rods with a diameter of $5 \mathrm{~mm}$ are used as metallic electrodes. One of them is placed in quartz ampoule with the diaphragm with a diameter of $2 \mathrm{~mm}$. The plasma zone is created in water vapor bubble at the diaphragm. The solution volume was $500 \mathrm{~mL}$. Dye's mixture solution was pumped to the reactor through a photometric bath using a peristaltic pump. The circulation rate was $80 \mathrm{~mL} \mathrm{~min}^{-1}$. After treatment, the solution is collected for further analysis. Time of plasma treatment was $25 \mathrm{~min}$.

Table 1 Molecular structure of dyes

Name Chemical structure


Fig. 1 Scheme of experimental setup for in situ study of decolorization process $1-a$ solution of mixture dyes, 2 quartz ampoule, 3-graphite electrodes, 4-spectrophotometric bath, 5-photometric probes, 6-control unit, 7-PC, and 8-plasma zone

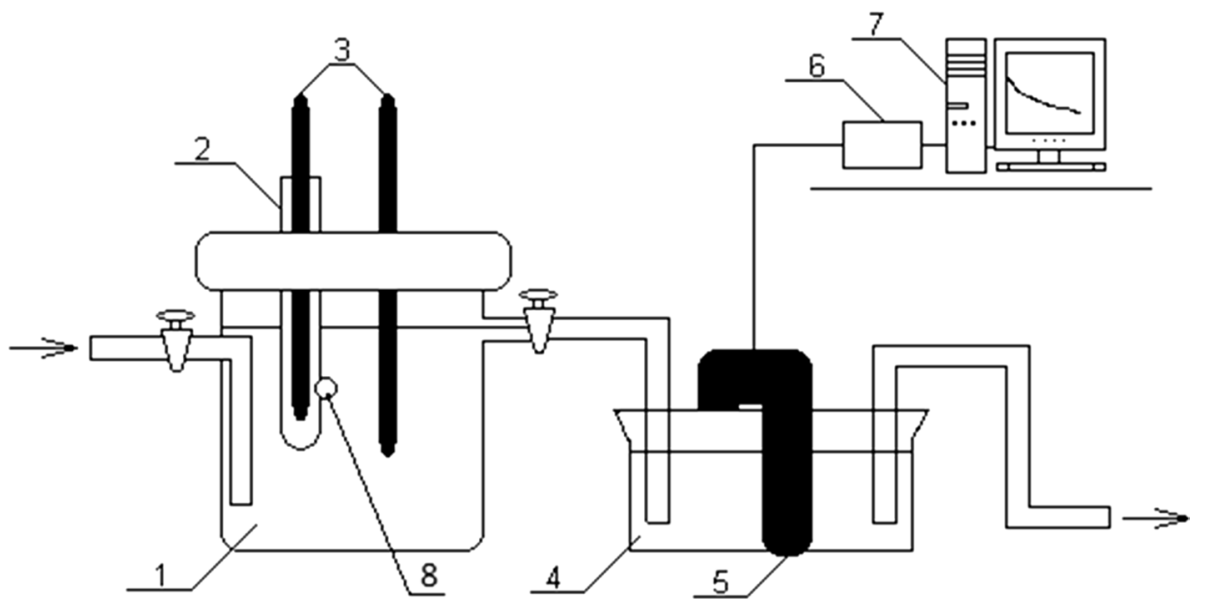

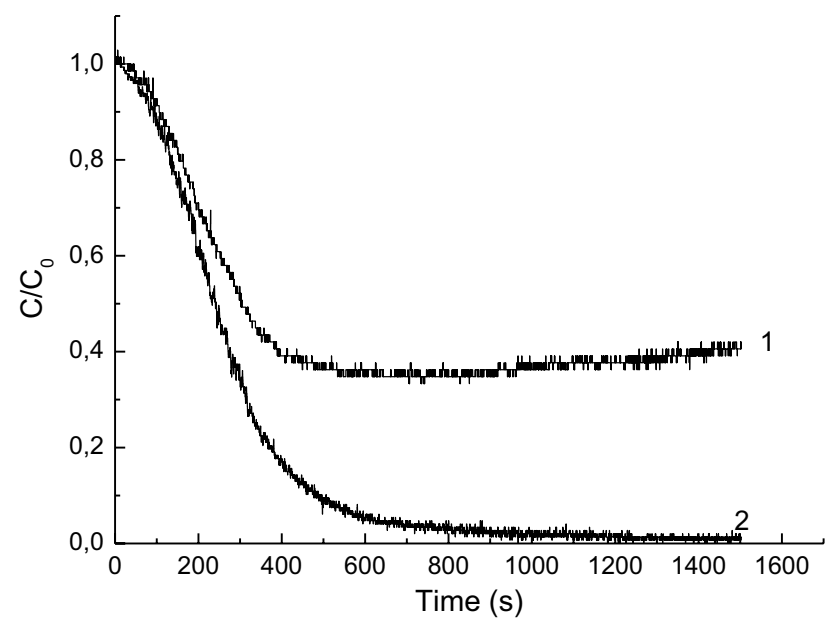

Fig. 2 Kinetic curves absorption intensity during plasma treatment. 1-Direct Blue dye and 2-Rhodamine 6G dye in the experiments with ratio of Rh6G to DB of 1:1.5

\subsection{Methods of analysis}

The mixtures of dyes were prepared by dissolving two dyes in deionized water. The concentration of Direct Blue 1 dye varied from 4 to $12 \mathrm{mg} / \mathrm{L}$. The concentration of Rhodamine $6 \mathrm{G}$ was constant and equaled to $4 \mathrm{mg} / \mathrm{L}$.

The concentration of dyes during plasma treatment was determined according to the Beer-Lambert law using photometric probes with wavelength $530 \mathrm{~nm}$ and $595 \mathrm{~nm}$ (Laboratory "L-micro", Russia). The typical curves are depicted in Fig. 2.

The whole absorption spectra in the range of wavelength $190-750 \mathrm{~nm}$ were registered by UV-Vis absorption spectroscopy (SF 104, Akvilon, Russia) before and after plasma treatment. The quartz cuvettes with a length of $10 \mathrm{~mm}$ were used.
The concentrations of oxidizers were determined using the following methods. The $\mathrm{H}_{2} \mathrm{O}_{2}$ is detected using the reaction of hydrogen peroxide with $\mathrm{KI}$ in the presence of $\mathrm{NH}_{4} \mathrm{MoO}_{4}$ as the catalyst in an acid medium. The total concentration of oxidizers was determined by using the reaction with potassium permanganate. The products of the degradation process were analyzed by high-performance liquid chromatography (Gilson 302) and gas chromatography mass spectrometry (Shimadzu GCMS QP2010 Ultra).

For investigating an effect of the initial temperature of a solution on dye's mixture decolorization in the absence of plasma, series experiments were carried out in the range of $10-80^{\circ} \mathrm{C}$. No changes in absorbance spectra are observed for $25 \mathrm{~min}$. It means that dye's mixture solution is thermally stable.

To determine the role of hydrogen peroxide in the destruction mechanism of dyes, the experiments were carried out without plasma treatment. $2 \mathrm{~mL}$ of $\mathrm{H}_{2} \mathrm{O}_{2}(30 \%)$ is added to the solutions of dye's mixture and single dyes every $5 \mathrm{~min}$. The changes of absorbance for both dyes are registered by photometric probes.

The acidity and conductivity of solutions were registered by $\mathrm{pH}$ meter I-160 (Akvilon, Russia) and conductivity meter InoLab (WTW, Germany) before and after plasma treatment.

\subsection{Calculations}

Decolorization efficiency is defined as a percent decrease in absorbance according to Eq. (1):

$\alpha=\frac{A_{0}-A}{A_{0}} \times 100 \%$, 
where $A_{0}$ and $A$ are the absorbance intensity of the initial dye's mixture solution and after plasma treatment (25 min).

The Rh6G and DB 1 decolorization rates are estimated according to Eq. (2):

$r=\frac{\mathrm{d} C}{\mathrm{~d} t}{ }_{t \rightarrow 0}$.

The effect of temperature on the decolorization rates for Rhodamine $6 \mathrm{G}$ and the Direct Blue 1 was studied. The first-order rate constants were estimated for all temperature range $\left(10-80^{\circ} \mathrm{C}\right)$, and the Arrhenius equation in the linear form (Eq. 3) is used to estimate the activation energy of the Rh6G and DB1 destruction reaction in the solution of mixture dyes by plasma treatment.

$\ln k=\ln A-\frac{E_{\mathrm{a}}}{R T^{\prime}}$

where $E_{\mathrm{a}}$ is activation energy $\left(\mathrm{J} \mathrm{mol}^{-1}\right), A$ is frequency factor $\left(\mathrm{s}^{-1}\right), R$ is gas constant $\left(8.31 \mathrm{~J} \mathrm{~K}^{-1} \mathrm{~mol}^{-1}\right)$, and $T$ is an absolute temperature $(\mathrm{K})$.

The energy yield is calculated using Eq. (4):

$G=\frac{\left(C_{0}-C_{f}\right) \cdot V}{P \cdot t}(\mathrm{~g} / \mathrm{kWh})$

where $C_{0}$ is an initial concentration of a dye, $C_{f}$ is a residual concentration of dye after plasma treatment, $V$ is volume of a solution, $P$ is an input power, and $t$ is the time of treatment.

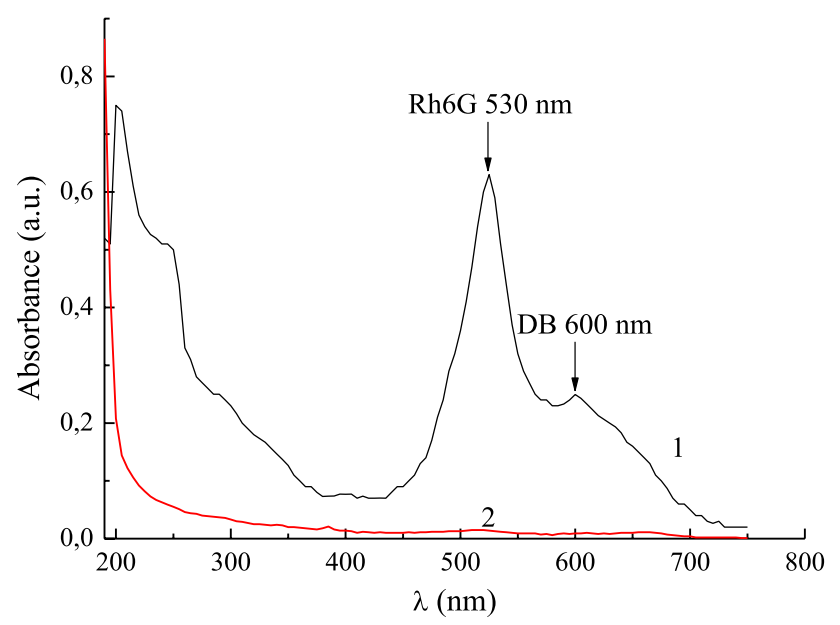

Fig. 3 Absorption spectra of dyes mixture solution before (1) and after (2) plasma treatment. The concentration of both dyes is $4 \mathrm{mg} / \mathrm{L}$

\section{SN Applied Sciences}
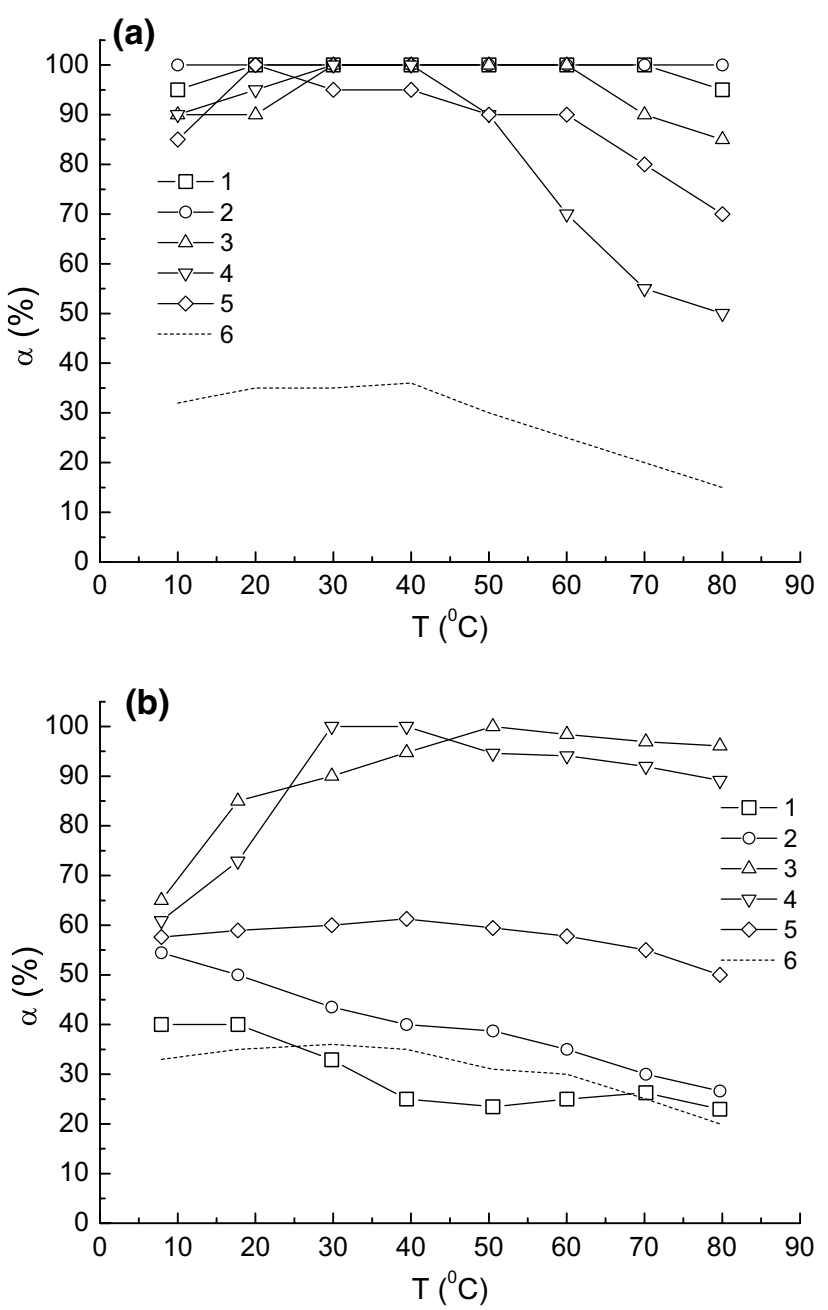

Fig. 4 Effect of initial temperature on the decolorization efficiency for Rhodamine 6G (a) and Direct Blue (b) in their mixture at concentration of DB: $1-4 \mathrm{mg} / \mathrm{L}, 2-6 \mathrm{mg} / \mathrm{L}, 3-8 \mathrm{mg} / \mathrm{L}, 4-10 \mathrm{mg} / \mathrm{L}$, $5-12 \mathrm{mg} / \mathrm{L}$, and 6-single dye. Concentration of Rh6G is $4 \mathrm{mg} / \mathrm{L}$

\section{Results and discussion}

\subsection{Decolorization efficiency $(a)$}

The absorbance spectra of untreated and treated solutions of the dye's mixture are depicted in Fig. 3. The plasma discharge treatment leads to the disappearance of peaks for both dyes in the visible region and peaks in the range of 200-300 nm. However, the peak at $\lambda<200 \mathrm{~nm}$ has appeared. It can be a peak at $190 \mathrm{~nm}$ that corresponded to oxalic acid [43].

The $\mathrm{pH}$ of treated solution did not change significantly $(\Delta \mathrm{pH}=-0.5)$, whereas the conductivity of these solutions increased (up to $190 \mu \mathrm{S} \mathrm{cm}^{-1}$ ). This can be connected with appearance of anions in solutions. One can assume that an increase in the electrical conductivity of the solution 
may prevent the complete decomposition of the Direct Blue dye (Fig. 2).

The effect of the conductivity of the solution on the generation/accumulation of reactive species or the destruction of organic compounds in aqueous solutions under the action of non-equilibrium plasma is a very interesting and still open question. Thorough research is required to answer this question. In the works [25, 30], it was found that an increase in the electrical conductivity of the solution leads to a decrease in the destruction efficiency of the dye. To date, we can only assume that an increase in electrical conductivity leads to an increase in the channels of expenditure of reactive species (competing reactions).

The effect of the initial temperature of a solution and concentration of DB dye on decolorization efficiency of two dyes in their mixture is presented in Fig. 4. The obtained results showed that the temperature range of $30-60{ }^{\circ} \mathrm{C}$ is optimal for reaching the maximal value of decolorization efficiency for Rhodamine $6 \mathrm{G}$ in all ranges of the dye's ratios. It should note that the maximal values of $a$ for both dyes were reached at $T=50^{\circ} \mathrm{C}$ at concentration of blue dye of $8 \mathrm{mg} / \mathrm{L}$ and in the range of $30-40{ }^{\circ} \mathrm{C}$ at the concentration of DB of $6 \mathrm{mg} / \mathrm{L}$. For the comparison, data on the efficiency of decolorization of single dye (not in a mixture) at plasma treatment are also given (curve 6 in Fig. 4). The dye concentration was $4 \mathrm{mg} / \mathrm{L}$. One can see the decolorization efficiency for Rhodamine $6 \mathrm{G}$ is increased by three times. In the case of an azo dye with the same concentrations, the effect is noticeable only at low temperatures.

\subsection{Rate of destruction}

The rates of destruction were estimated by numerical differentiation of the kinetic curves of decolorization. Our results showed that the rates changed with the time of treatment. The same behavior was found in our previous studies [39-41]. It means that the serial-parallel reactions pass in the solution and finally we have some effective process of destruction. As known, the ignition of electrical discharge inside or above the aqueous solution initiates the formation of reactive species in a solution. We suggested that the dyes react with reactive species at the initial stage of plasma action. And then, not only reactive species but intermediates take part in the destruction process. In this case, the values of the rate of destruction at the initial time of treatment were chosen for the comparison of the rate of destruction for both dyes at various experimental conditions.

Our experimental results showed that the initial rate of destruction of the Direct Blue 1 dye increases with the concentration in a solution (Fig. 5). It is the following data of

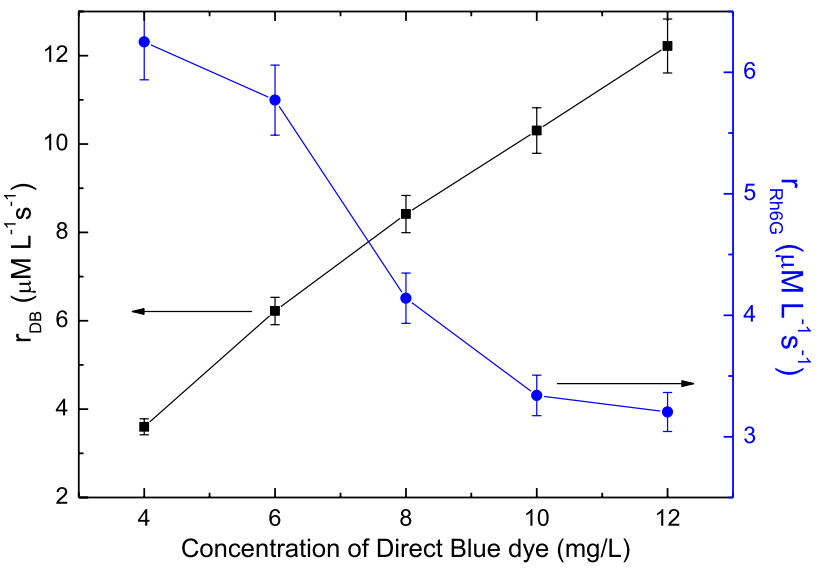

Fig. 5 Effect of DB1 dye concentration on the destruction rates for Rhodamine $6 \mathrm{G}\left(\mathrm{r}_{\mathrm{Rh} 6 \mathrm{G}}\right)$ and Direct Blue $\left(\mathrm{r}_{\mathrm{DB}}\right)$ dyes at initial temperature of solution of $20^{\circ} \mathrm{C}$
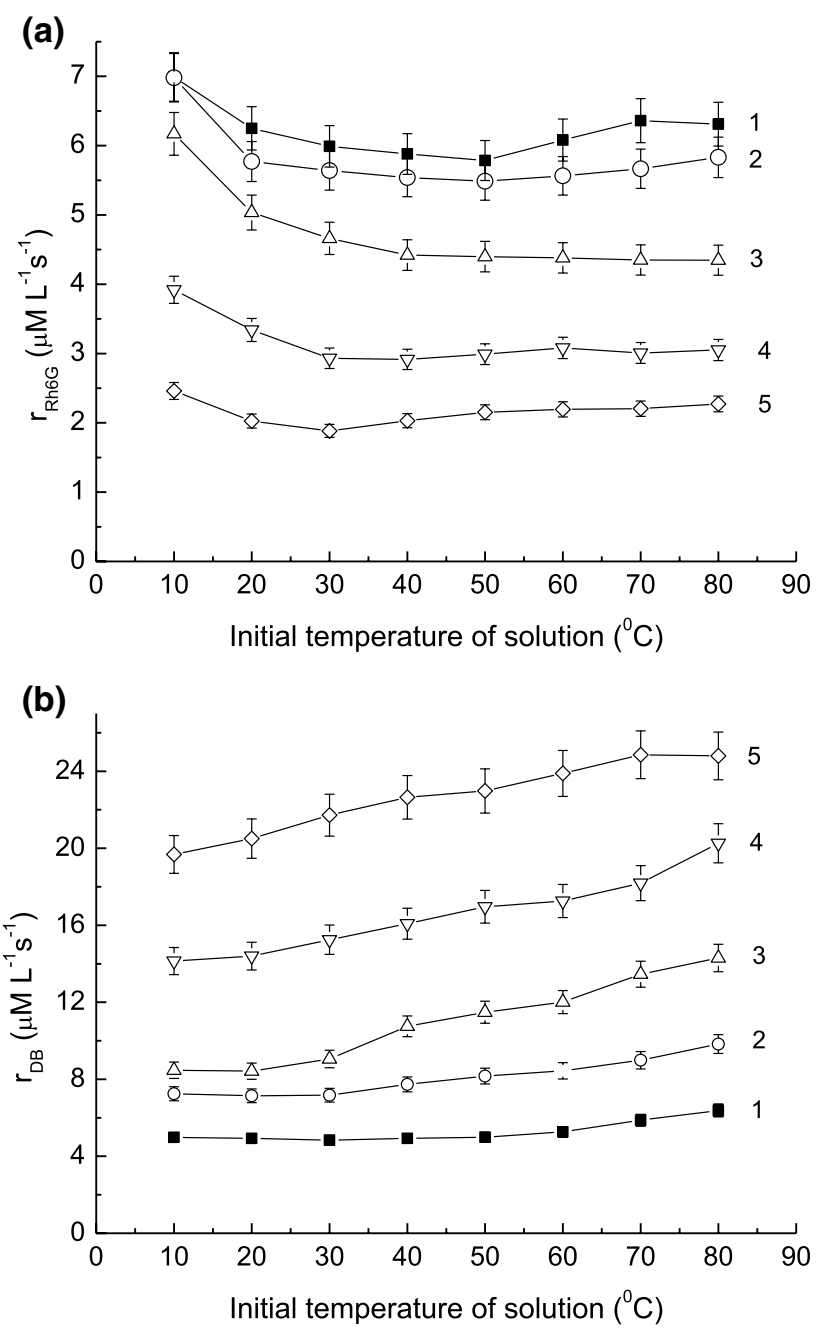

Fig. 6 Destruction rates for Rhodamine 6G (a) and for Direct Blue 1 dye (b) as a function of an initial temperature of dye's mixture solution at different concentrations of DB1: $1-4 \mathrm{mg} / \mathrm{L}, 2-6 \mathrm{mg} / \mathrm{L}$, $3-8 \mathrm{mg} / \mathrm{L}, 4-10 \mathrm{mg} / \mathrm{L}$, and $5-12 \mathrm{mg} / \mathrm{L}$. The concentration of $\mathrm{Rh} 6 \mathrm{G}$ is $4 \mathrm{mg} / \mathrm{L}$ 
work [44], where authors found that in the range of small concentration of dye (33-150 mg/L) rate of destruction is linear with an increase in concentration. In the case of Rh6G, the increasing concentration of the DB1 dye in a solution leads to decreasing the rate of destruction. It is obviously due to competing reactions with the participation of the reactive species.

\subsection{Effect of initial temperature}

For Rhodamine $6 \mathrm{G}$ dye, in the temperature range of $10-30^{\circ} \mathrm{C}$, the increasing temperature induces the decrease in the destruction rate values (Fig. 6a). It can be connected with the formation of a dimer form of this dye $[45,46]$. In the temperature range of $30-80^{\circ} \mathrm{C}$, increase in the initial temperature of a solution has little effect on the rate of the destruction. It is possible only in the case that the reaction passes into an intradiffuse zone of a solution. As known, the reactive species are formed in the small volume of a solution near to plasma zone [47]. The interaction of the hydroxyl radicals and molecule of Rh6G occurs in this small area, which can relate to an intradiffuse zone.

The formation of hydrogen peroxide can occur as in the small volume of a solution near to plasma zone as well as in the bulk of a solution (diffuse zone). In this case, the temperature of solution effects on the rate of the interaction as reflected for Direct Blue 1, the increase in initial temperature of a solution leads to the growth in the rate of destruction (Fig. 6b).

\subsection{A possible mechanism of the destruction}

When the plasma is ignited above or inside aqueous solutions, the reactive species, such as hydrogen atoms, hydroxyl radicals, hydrated electrons, and oxygen, are formed in a liquid. The main oxidative agent is $\mathrm{OH}$ radical, which leads to the destruction of organic dyes [48-50]. It was assumed that the hydroxyl radicals are responsible for the destruction of both dyes. However, analysis of kinetic curves for Rh6G and DB1 showed that the reactive species that are responsible for destruction might be different. According to data of kinetics of the decolorization, the process for Rhodamine $6 \mathrm{G}$ is faster than for Direct Blue 1. This means that the destruction process may occur with primary reactive species, such as $\mathrm{OH}$ radicals for Rh6G. It is possible because of the high rate constant reaction of hydroxyl radical with $\mathrm{Rh} 6 \mathrm{G}\left(1.1 \times 10^{10} \mathrm{~L} \mathrm{~mol}^{-1} \mathrm{~s}^{-1}\right)[51]$.

There are a lot of publications related to the study of formation and accumulation of hydrogen peroxide in aqueous solution by plasma treatment which is collected in the review Locke and Shih [52]. Thus, $\mathrm{H}_{2} \mathrm{O}_{2}$ can be the reactive agent responsible for the destruction process also.

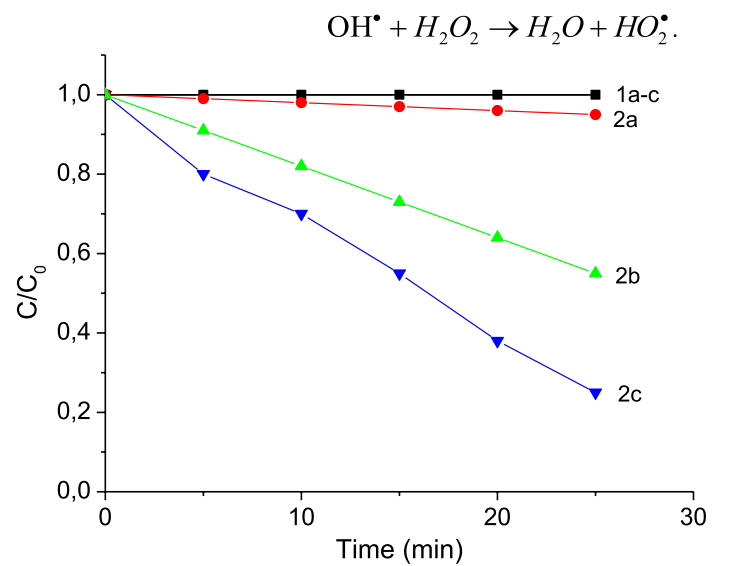

Fig. 7 Kinetic curves of destruction for Rhodamine 6G (1) and Direct Blue 1 (2) dyes at next conditions: (a) adding hydrogen peroxide to solution of single dye without plasma, (b) joint action of plasma and $\mathrm{H}_{2} \mathrm{O}_{2}$ on the solution of single dye, and (c) addition of $\mathrm{H}_{2} \mathrm{O}_{2}$ to solution of dyes mixture

A series of experiments were carried out on the effect of the addition of hydrogen peroxide on the decomposition of Rhodamine $6 \mathrm{G}$ and Direct Blue 1 dye individually and in a mixture without plasma and with combined action (Fig. 7). The results showed that the Rh6G is very resistant to hydrogen peroxide (curves 1 in Fig. 7). The absence of any effect with the combined action of hydrogen peroxide and plasma on the solution of a single dye is explained by the occurrence of a competing reaction involving the hydroxyl radical and $\mathrm{H}_{2} \mathrm{O}_{2}$ [51].

$\mathrm{OH}+\mathrm{H}_{2} \mathrm{O}_{2} \rightarrow \mathrm{H}_{2} \mathrm{O}+\mathrm{HO}_{2}$.

In the case of the azo dye, the addition of hydrogen peroxide without the plasma treatment of single-dye solution leads to $5 \%$ decomposition (curve $2 \mathrm{a}$ in Fig. 7). The combined effect of $\mathrm{H}_{2} \mathrm{O}_{2}$ and plasma causes $45 \%$ of DB1 destruction (curve $2 \mathrm{~b}$ in Fig. 7). The greatest effect was registered in a mixture of dyes with the addition of hydrogen peroxide (curve $2 \mathrm{c}$ in Fig. 7).

We supposed that $\mathrm{HO}_{2}$ or $\mathrm{H}_{2} \mathrm{O}_{2}$ reactive species are responsible for the destruction of DB dye. The dimerization of hydroxyl radicals is not one of the ways for the production of hydrogen peroxide. Moreover, the results of chemical analysis showed that the concentration of total oxidizers is $0.3 \mathrm{mM} / \mathrm{L}$, whereas the concentration of $\mathrm{H}_{2} \mathrm{O}_{2}$ is $0.225 \mathrm{mM} / \mathrm{L}$ and concentration of ozone did not exceed $0.05 \mathrm{mM} / \mathrm{L}$. This means that about $0.025 \mathrm{mM} / \mathrm{L}$ of long-lived (stable) compounds with oxidizing properties is present in the solution.

The results of GC/MS analysis in positive electrospray mode showed a dominant signal at $m / z=319$ corresponds to 4-amino-5-hydroxynaphthalene-1,3-disulfonic acid 


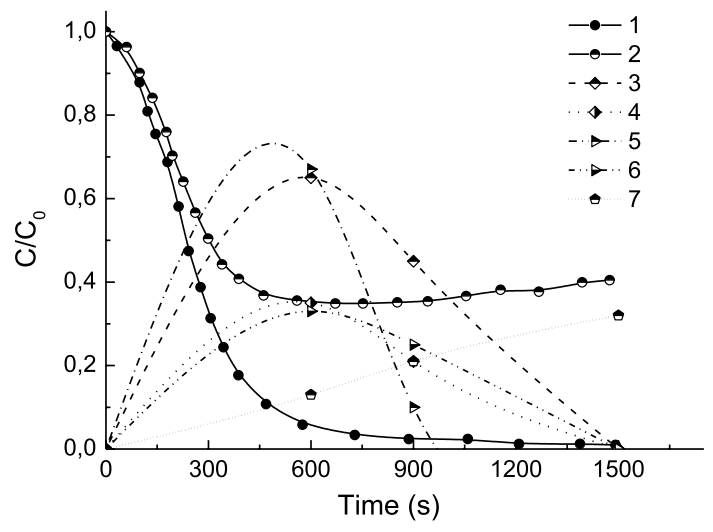

Fig. 8 Rhodamine $6 \mathrm{G}$ and Direct Blue 1 by-products evolution during plasma treatment: 1-Rh6G, 2-DB1, 3-3-aminophenol, 4-benzaldehyde, 5-1,1'dimethoxybenzidine, 6-SS acid, and 7oxalic acid

(SS acid) as the main by-product. Additional peaks were registered at $m / z=244$ (dimethoxybenzidine), $m / z=123$ (aminoquinone), $m / z=109$ (aminophenol), and $m / z=106$ (benzaldehyde). The mass spectra of solution after $25 \mathrm{~min}$ of treatment showed the significant decrease in these peaks. This means that products of partial destruction of the organic dyes are also oxidizers and could take part in the oxidation processes. This is confirmed by the results of
LC (Fig. 8). The results of liquid chromatography analysis of solution after plasma treatment showed the presence of the oxalic acid and inorganic anions, such as nitrate, sulfate, and chloride ions.

Based on the results of GC/MS analysis, we suggested the possible chemical pathways for destruction processes of dyes. In the case of Rhodamine $6 \mathrm{G}$, the interaction between hydroxyl radicals and the molecule of dye leads to the break of the oxygen bridge that is responsible for the intensity of the color and removal of an ether group. Further destruction process leads to the production of an aminoquinone (1) in Scheme 1), which also has a high oxidizing ability. The formation of aminophenol (2) and benzaldehyde (3) is possible (Scheme 1).

In the case of Direct Blue 1, the reactive species react with the molecule's aminonaphthol part. It leads to the destruction of double nitric bonds, which are responsible for the color of this class of dyes. We suggest the formation of SS acid (4) and dimethoxybenzidine (5) which are transparent where the visible spectrum region occurs (Scheme 2).

\subsection{Activation energy}

Creating a plant for the treatment, the appropriate physical-chemical parameters are needed. In particular, the
Scheme 1 Possible destruction pathway of Rhodamine $6 \mathrm{G}$ and formation of by-products<smiles></smiles>

Scheme 2 Possible degradation pathway of Direct Blue dye<smiles>COc1cc(-c2ccc(N=Nc3ccc(-c4ccc(N=Nc5ccc6c(S(=O)(=O)O)cc(S(=O)(=O)O)c(N)c6c5O)c(OC)c4)cc3OC)c(OC)c2)ccc1N</smiles>

(4) 
Table 2 Apparent activation energy values

\begin{tabular}{lrl}
\hline \multirow{2}{*}{$\begin{array}{l}\text { Concentration } \\
\text { of DB1 }(\mathrm{mg} / \mathrm{L})\end{array}$} & \multicolumn{2}{l}{$E_{\mathrm{a}}\left(\mathrm{kJ} \mathrm{mol} \mathrm{mo}^{-1}\right)$} \\
\cline { 2 - 3 } & $\mathrm{Rh6G}$ & $\mathrm{DB} 1$ \\
\hline 4 & 6.01 & 14.99 \\
6 & 13.72 & 14.5 \\
8 & 17.54 & 13.82 \\
10 & 31.26 & 7.75 \\
12 & 33.29 & 6.28 \\
\hline
\end{tabular}

activation energy is one of a parameter that can predict the behavior of a reaction. In the case of single-step reactions, the value of $E_{\mathrm{a}}$ is determined easily. In the case of complex reactions, the activation energy value can estimate as an apparent $E_{\mathrm{a}}$.

We took into account the fact that the efficacy of dye's destruction decreased at $T>50^{\circ} \mathrm{C}$. The estimation of energy activation values is carried out in the temperature range of $10-50{ }^{\circ} \mathrm{C}$ using Eq. (3). Calculated values are presented in Table 2. The low values of $E_{\mathrm{a}}\left(6-17 \mathrm{~kJ} \mathrm{~mol}^{-1}\right)$ attribute to reaction with radicals $\left(\mathrm{OH}^{-}\right.$and $\left.\mathrm{HO}_{2}\right)$ [51]. Obtained values are comparable with published data [53-59].

Decreasing activation energy values for Direct Blue 1 dye means that the substance/catalyst is present in the solution which eases the destruction process. It could be aminoquinone. And reverse, increasing $E_{\mathrm{a}}$ for Rhodamine $6 \mathrm{G}$ with growth concentration of blue dye means that the inhibitor appeared in the solution. This can be one of the products of DB1 destruction process, for example oxalic acid. To check these assumptions, the two series of experiments were carried out without plasma treatment. In the first experiment, synthesized aminoquinone is added to dyes mixture at a ratio of dyes of $1: 1$ at $20^{\circ} \mathrm{C}$. The obtained results showed that the decolorization efficiency of Direct Blue 1 dye is increased to $85 \%$ and the initial rate of destruction is increased by 1.4 times. This means that the by-product of Rhodamine $6 \mathrm{G}$ is a catalyst for the destruction process of DB1. In the other experiments, the oxalic acid was added to dyes mixture at the same conditions. The initial rate of destruction for Rh6G is decreased by $25 \%$. And it means that oxalic acid is an inhibitor of the destruction process of Rh6G. The obtained results are consistent with previously published data $[58,60]$, which were found that oxalic acid is an inhibitor of the decomposition reaction of the dye Rhodamine $B$.

\subsection{Energy yield}

The energy efficiency (yield) is a basic parameter of suggesting a method for cleaning. This parameter is determined as the amount of destroyed dye divided by the consumed energy $(G)$. The results of calculations using

Eq. (4) are presented in Table 3. Despite the decrease in the efficiency of azo dye bleaching, the values of $G$ become constant at concentrations of 8-12 mg/L. Our results are in qualitative agreement with the data of work 42 . The values of the energy yield are higher for dyes taken in excess.

Using the methylene blue dye as an example, we can trace that different values of $G$ are obtained using the same dye concentration and even the same electrode configuration system (the same type of electric discharge). It should be noted that in the work [16], to accelerate the decomposition process, the experiments are carried out in an oxygen atmosphere, whereas the authors of [21] used the addition of iron(II) ions. According to the energy yield values, conducting the experiments in an atmosphere of $\mathrm{O}_{2}$ is more efficient.

An analysis of the data presented showed that the energy the yields are determined by the concentration of the dye, the degree of its decomposition, and the energy input. The combination of low-temperature plasma with the addition of hydrogen peroxide $[12,30,31]$, iron(II) ions $[12,21,25,30,35]$, bubbling with ozone [10, 14], or conducting experiments in an oxygen atmosphere [12, 16, $17,33,38]$, as well as the use of nanoparticles [14, 24, 32], accelerates the destruction processes and increases $G$.

The following point should be noted. When calculating the value of $G$, it is assumed that all the input energy is spent on the destruction processes. According to works $[47,61,62]$, most of the input energy of $55-60 \%$ is consumed in heating the solution. Around $30 \%$ is distributed among radiant energy, generation of acoustic waves, and initiation of the chemical processes (23\%) [47]. As was mentioned above, the increase in temperature of a solution does not induce the degradation processes. This means that most part of input energy does not take part in the destruction processes. In $[53,59,63,64]$, it was found that destruction of dyes occurs by acoustic waves and radiant energy (UV and visible radiation) also. Hence, only $1 / 3$ part of input energy is consumed in the destruction processes in a solution. In our case, this means that the $G$ values should be higher and it could be improved by applying at treatment facilities of a textile factory.

\section{Conclusions}

The application of the underwater AC diaphragm discharge plasma for the destruction of a mixture of two dyes was found to be effective. The efficiency of the destruction process was proved by UV-Vis spectra and HPLC-GC/ MS analysis, which indicated that the destruction process occurs via the formation of the by-products. Experimentally proven that the Rhodamine $6 \mathrm{G}$ degradation byproduct (aminoquinone) is a catalyst for the Direct Blue 
Table 3 Energy yields of dyes by electric discharge treatment

\begin{tabular}{|c|c|c|c|c|}
\hline Dye & $\begin{array}{l}\text { Concentration } \\
(\mathrm{mg} / \mathrm{L})\end{array}$ & Decolorization (\%) & $G(\mathrm{~g} / \mathrm{kWh})$ & References \\
\hline \multicolumn{5}{|l|}{ Solution of mono dye } \\
\hline \multirow[t]{6}{*}{ Methylene Blue } & 50 & 50 & 1.3 & {$[21]$} \\
\hline & 4.8 & 52 & 0.035 & {$[28]$} \\
\hline & 10 & 90 & 4.6 & {$[12]$} \\
\hline & 40 & 93 & 0.29 & [13] \\
\hline & 50 & 95 & 57 & [16] \\
\hline & 13.25 & 100 & 30 & [10] \\
\hline Acid Black 52 & 4.6 & 100 & 0.008 & {$[35]$} \\
\hline \multirow[t]{2}{*}{ Acid Blue 25} & 10 & 50 & 0.0012 & {$[29]$} \\
\hline & 30 & 50 & 2.4 & {$[23]$} \\
\hline \multirow[t]{2}{*}{ Acid Orange 7 (Orange II) } & 20 & 50 & 5.61 & {$[27]$} \\
\hline & 1000 & 41.5 & 251 & [19] \\
\hline Acid Red 4 & 20 & 100 & 4.36 & {$[20]$} \\
\hline Acid Red 27 & 10 & 100 & 5.5 & [14] \\
\hline Acid Red 88 & 25 & 100 & 2.88 & [17] \\
\hline Alizarin red S & 80.5 & 82 & 0.053 & {$[42]$} \\
\hline Aluminon & 50 & 65 & 0.14 & [37] \\
\hline Amaranth azo dye & 50 & 95 & 4.17 & {$[15]$} \\
\hline Aniline Blue & 50 & 95 & 0.18 & [37] \\
\hline Azocarmine B & 50 & 100 & 0.28 & {$[37]$} \\
\hline Bismark Brown & 50 & 96 & 0.245 & {$[37]$} \\
\hline \multirow[t]{2}{*}{ Congo Red } & 50 & 61.8 & 1.39 & {$[30]$} \\
\hline & 1000 & 56.41 & 179 & [19] \\
\hline Crystal Violet & 4.1 & 100 & 4.23 & {$[38]$} \\
\hline Direct Blue 1 & 4 & 30 & 0.005 & This work \\
\hline Indigo Carmine & 30 & 40 & 0.4 & [36] \\
\hline Malachite Green & 50 & 96 & 0.255 & [37] \\
\hline Methyl Orange & 40 & 82 & 0.52 & [13] \\
\hline Methyl Red & 40 & 90 & 0.57 & [13] \\
\hline Methyl Yellow & 40 & 60 & 0.38 & [13] \\
\hline Naphthol Green B & 50 & 50 & 0.07 & [37] \\
\hline Naphthol Blue Black & 1000 & 53.25 & 215 & [19] \\
\hline \multirow[t]{2}{*}{ Orange G } & 1000 & 50.81 & 215 & [19] \\
\hline & 100 & 82 & 0.066 & {$[42]$} \\
\hline Phenol Red & 40 & 93 & 1.17 & [13] \\
\hline Pyrocatechol Violet & 50 & 70 & 0.145 & [37] \\
\hline Reactive Blue 4 & 20 & 100 & 4.36 & {$[20]$} \\
\hline \multirow[t]{2}{*}{ Reactive Blue 19} & 20 & 87 & 0.027 & {$[31]$} \\
\hline & 100 & 100 & 8 & [33] \\
\hline Rhodamine 6G & 4 & 35 & 0.009 & This work \\
\hline Sulfanilic acid azochromotrop & 50 & 97 & 0.18 & [37] \\
\hline Stilbazo & 50 & 70 & 0.15 & [37] \\
\hline \multicolumn{5}{|l|}{ Mixture of dyes } \\
\hline \multirow[t]{2}{*}{ Alizarin red $\mathrm{S}+$ Orange $\mathrm{G}$} & $322+100$ & 80 for both dyes & 0.206 (AR) & {$[42]$} \\
\hline & & & 0.064 (OG) & \\
\hline
\end{tabular}


Table 3 (continued)

\begin{tabular}{|c|c|c|c|c|}
\hline Dye & $\begin{array}{l}\text { Concentration } \\
\text { (mg/L) }\end{array}$ & Decolorization (\%) & $G(\mathrm{~g} / \mathrm{kWh})$ & References \\
\hline \multirow[t]{14}{*}{ Rhodamine $6 \mathrm{G}+$ Direct Blue 1} & \multirow[t]{2}{*}{$4+8$} & 25 (Rh6G) & 0.032 & \multirow[t]{2}{*}{ [41] } \\
\hline & & 20 (DB1) & 0.051 & \\
\hline & \multirow[t]{2}{*}{$0.4+0.4$} & 100 (Rh6G) & 0.013 & \multirow[t]{2}{*}[40]{} \\
\hline & & $58(\mathrm{DB} 1)$ & 0.007 & \\
\hline & \multirow[t]{2}{*}{$4+4$} & 100 (Rh6G) & 0.13 & \multirow[t]{10}{*}{ This work } \\
\hline & & 40 (DB1) & 0.051 & \\
\hline & \multirow[t]{2}{*}{$4+6$} & 100 (R6G) & 0.13 & \\
\hline & & 50 (DB1) & 0.096 & \\
\hline & \multirow[t]{2}{*}{$4+8$} & 90 (R6G) & 0.12 & \\
\hline & & 85 (DB1) & 0.22 & \\
\hline & \multirow[t]{2}{*}{$4+10$} & 95 (R6G) & 0.12 & \\
\hline & & 73 (DB1) & 0.23 & \\
\hline & \multirow[t]{2}{*}{$4+12$} & 100 (R6G) & 0.13 & \\
\hline & & 60 (DB1) & 0.23 & \\
\hline
\end{tabular}

1 destruction process and oxalic acid is an inhibitor for the destruction of Rh6G. It is confirmed by the estimated energy activation of the destruction process for both dyes. The final compounds in a solution are oxalic acid and inorganic anions.

The results showed that the maximal decolorization efficiency $(100 \%)$ is reached in the temperature range of $30-50{ }^{\circ} \mathrm{C}$. This temperature range is an important parameter in the further application of this method to the real textile wastewater. It allows using the underwater diaphragm discharge with real wastewater without preliminary heat, since the real sewage of textile factories has a temperature around $30-40{ }^{\circ} \mathrm{C}$. The presence of a catalyst in the system allows optimizing the conditions for cleaning. The formation of aminoquinone occurs for the first minutes of plasma treatment, and maximal concentration can reach for $10 \mathrm{~min}$. The presence of catalyst and by-products can initiate the destruction process after plasma off (so-called post-effect). This allows reducing processing time and decreases energy costs. Based on these results, the next work is expected to develop the module prototype for cleaning textile wastewater in a sump of the textile factory.

Acknowledgements The liquid chromatography and gas chromatography mass spectrometry analysis was carried out using by High Performance-Liquid Chromatograph Gilson 302 (France) and Shimadzu GCMS QP2010 Ultra (Shimadzu Europa $\mathrm{GmbH}$ ) at the Interdepartmental Laboratory of Gas, Mass-Spectrometry, and EPR Spectrometry at the Ivanovo State University of Chemistry and Technology.

\section{Compliance with ethical standards}

Conflict of interest On behalf of all authors, the corresponding author states that there is no conflict of interest.

\section{References}

1. Ghanbari F, Moradi M (2015) A comparative study of electrocoagulation, electrochemical Fenton, electro-Fenton and peroxicoagulation for decolorization of real textile wastewater: electrical energy consumption and biodegradability improvement. J Environ Chem Eng 3:499-506

2. Brillas E, Martínez-Huitle CA (2015) Decontamination of wastewaters containing synthetic organic dyes by electrochemical methods. An updated review. Appl Catal B Environ 166:603-643

3. Nidheesh PV, Zhou M, Oturan MA (2018) An overview on the removal of synthetic dyes from water by electrochemical advanced oxidation processes. Chemosphere 197:210-227

4. Lafi R, Gzara L, Lajimi RH, Hafiane A (2018) Treatment of textile wastewater by a hybrid ultrafiltration/electrodialysis process. Chem Eng Process Process Intensif 132:105-113

5. Katheresan V, Kansedo J, Lau SY (2018) Efficiency of various recent wastewater dye removal methods: a review. J Environ Chem Eng 6:4676-4697

6. Jiang B, Zheng J, Qui S, Wu M, Zhang Q, Yan Z, Xue Q (2014) Review on electrical discharge plasma technology for wastewater remediation. Chem Eng J 236:348-368

7. Zhang H, Ma D, Qiu R, Tang Y, Du C (2017) Non-thermal plasma technology for organic contaminated soil remediation: a review. Chem Eng J 313:157-170

8. Thagard SM, Locke BR (2018) Electrical discharge plasma for water treatment. In: Stefan MI (ed) Advanced oxidation processes for water treatment: fundamentals and applications, 1st edn. IWA Publishing, London, pp 493-533 
9. Magureanu M, Bradu C, Parvulescu VI (2018) Plasma processes for the treatment of water contaminated with harmful organic compounds. J Phys D Appl Phys 51:313002

10. Malik MA, Ghaffar A, Ahmed K (2002) Synergistic effect of pulsed corona discharges and ozonation on decolourization of methylene blue in water. Plasma Sources Sci Technol 11:236

11. Stará Z, Krcma F (2005) Degradation of organic dyes versus $\mathrm{H}_{2} \mathrm{O}_{2}$ generation during the DC diaphragm discharge treatment in water solutions. Acta Phys Slovaca 55:515-519

12. Grabowski LR, Van Veldhuizen EM, Pemen AJM, Rutgers WR (2007) Breakdown of methylene blue and methyl orange by pulsed corona discharge. Plasma Sources Sci Technol 16:226

13. Magureanu M, Mandache NB, Parvulescu VI (2007) Degradation of organic dyes in water by electrical discharges. Plasma Chem Plasma Process 27:589-598

14. Mok YS, Jo JO, Lee HJ, Ahn HT, Kim JT (2007) Application of dielectric barrier discharge reactor immersed in wastewater to the oxidative degradation of organic contaminant. Plasma Chem Plasma Process 27:51-64

15. Mok YS, Ahn HT, Kim JT (2007) Treatment of dyeing wastewater by using positive pulsed corona discharge to water surface. Plasma Sci Technol 9:71

16. Magureanu M, Piroi D, Mandache NB, Parvulescu V (2008) Decomposition of methylene blue in water using a dielectric barrier discharge: optimization of the operating parameters. J Appl Phys 104:103306

17. Tang Q, Jiang W, Zhang Y, Wei W, Lim TM (2009) Degradation of azo dye acid red 88 by gas phase dielectric barrier discharges. Plasma Chem Plasma Process 29:291-305

18. Khlyustova AV, Subbotkina IN (2012) Kinetic regularities of methylene blue destruction process in different media under the impact of the diaphragm discharge plasma. High Temp Mater Process 16:71-79

19. Diono W, Machmudah S, Nagafuchi K, Sasaki M, Akiyama H, Goto M (2013) Oxidative decoloration of dyes by pulsed discharge plasma over a water surface under argon atmospheric. Trans Mater Res Soc Jpn 38:61-67

20. Kim SD, Jang DI, Lim BJ, Lee SB, Mok YS (2013) Degradation of synthetic dyeing wastewater by underwater electrical discharge processes. Plasma Sci Technol 15:659

21. Magureanu M, Bradu C, Piroi D, Mandache NB, Parvulescu V (2013) Pulsed corona discharge for degradation of methylene blue in water. Plasma Chem Plasma Process 33:51-64

22. Němcová L, Krčma F, Nikiforov A, Leys C (2014) Destruction of direct blue 106 dye in underwater discharge. J Phys Conf Ser 516:012008

23. Ghodbane $H$, Nikiforov $A Y$, Hamdaoui $O$, Surmont $P$, Lynen $F$, Willems G, Leys C (2014) Non-thermal plasma degradation of anthraquinonic dye in water: oxidation pathways and effect of natural matrices. J Adv Oxid Technol 17:372-384

24. Ghodbane H, Hamdaoui O, Vandamme J, van Durme J, Vanraes $P$, Leys $C$, Nikiforov AY (2015) Degradation of AB25 dye in liquid medium by atmospheric pressure non-thermal plasma and plasma combination with photocatalyst $\mathrm{TiO}_{2}$. Open Chem 13:25-331

25. Zhou Z, Zhang X, Liu Y, Ma Y, Lu S, Zhang W, Ren Z (2015) Treatment of azo dye (acid orange II) wastewater by pulsed highvoltage hybrid gas-liquid discharge. RSC Adv 5:71973-71979

26. Lee HD, Kim JO, Chung JW (2015) Degradation of methyl orange by pulsed corona discharge process in water. Desalin Water Treat 53:2767-2773

27. Wang T, Qu G, Ren J, Sun Q, Liang D, Hu S (2016) Organic acids enhanced decoloration of azo dye in gas phase surface discharge plasma system. J Hazard Mater 302:65-71
28. Mohammadi B, Ashkarran AA (2016) Cold atmospheric plasma discharge induced fast decontamination of a wide range of organic compounds suitable for environmental applications. J Water Process Eng 9:195-200

29. El-Tayeb A, El-Shazly A, Elkady M (2016) Investigation the influence of different salts on the degradation of organic dyes using non-thermal plasma. Energies 9:874

30. Haixia W, Zhi F, Tong Z, Chen L, Yanhua X (2016) Discoloration of congo red by rod-plate dielectric barrier discharge processes at atmospheric pressure. Plasma Sci Technol 18:500

31. AlHamad B, Al-Bastaki N (2016) Degradation of reactive blue 19 using advanced oxidation methods: gliding-arc plasma discharge. Desalin Water Treat 57:24352-24358

32. Koutahzadeh N, Esfahani MR, Arce PE (2016) Removal of acid black 1 from water by the pulsed corona discharge advanced oxidation method. J Water Process Eng 10:1-8

33. Sun Y, Liu Y, Li R, Xue G, Ognier S (2016) Degradation of reactive blue 19 by needle-plate non-thermal plasma in different gas atmospheres: kinetics and responsible active species study assisted by CFD calculations. Chemosphere 155:243-249

34. Shang K, Wang X, Li J, Wang H, Lu N, Jiang N, Wu Y (2017) Synergetic degradation of acid orange 7 (AO7) dye by DBD plasma and persulfate. Chem Eng J 311:378-384

35. Sánchez JV, Segundo CT, Palacios EM, Díaz AG, Romero PGR (2017) Degradation of textile dye AB 52 in an aqueous solution by applying a plasma at atmospheric pressure. IEEE Trans Plasma Sci 45:479-484

36. Hosano H, Sakugawa T, Akiyama H (2018) The role of pulse voltage amplitude on chemical processes induced by streamer discharge at water surface. Catalysts 8:213

37. Jamroz P, Dzimitrowicz A, Pohl P (2018) Decolorization of organic dyes solution by atmospheric pressure glow discharge system working in a liquid flow-through mode. Plasma Process Polym 15:1700083

38. Rahimpour M, Taghvaei H, Rahimpour MR (2019) Degradation of crystal violet in water solution using post discharge DBD plasma treatment: factorial design experiment and modeling. Chemosphere 232:213-223

39. Maximov A, Khlyustova A, Grosheva A (2012) Action of low-voltage underwater discharges on dyes solution mixtures. Izvestiya Vysshikh Uchebnykh Zavedenii. Seriya Khimiya i Khimicheskaya Tekhnologiya 55:40-43 (in rus)

40. Khlyustova AV, Maksimov Al, Panova DS (2013) Effect of electric discharges and oxidizing agents on aqueous solutions of a mixture of two organic dyes. Surf Eng Appl Electrochem 49:272-277

41. Khlyustova AV, Maximov Al (2013) Plasma-assisted oxidative degradation of organic dyes in solution by the joint action of underwater discharge and ozone. High Energy Chem 47:140-143

42. Merouani DR, Abdelmalek F, Taleb F, Martel M, Semmoud A, Addou A (2018) Plasma treatment by gliding arc discharge of dyes/dye mixtures in the presence of inorganic salts. Arab J Chem 8:155-163

43. Cruywagen JJ, Heyns JBB (1983) Determination of the dissociation constants of oxalic acid and the ultraviolet spectra of the oxalate species in $3 \mathrm{M}$ perchlorate medium. Talanta 30:97-200

44. Palma-Goyes RE, Guzmán-Duque FL, Peñuela G, González I, Nava JL, Torres-Palma RA (2010) Electrochemical degradation of crystal violet with BDD electrodes: effect of electrochemical parameters and identification of organic by-products. Chemosphere 81:26-32

45. Vogel R, Harvey M, Edwards G, Meredith P, Heckenberg N, Trau $M$, Rubinsztein-Dunlop H (2002) Dimer-to-monomer transformation of rhodamine $6 \mathrm{G}$ in aqueous PEO-PPO-PEO block copolymer solutions. Macromolecules 35:2063-2070 
46. Terdale S, Tantray A (2017) Spectroscopic study of the dimerization of rhodamine $6 \mathrm{G}$ in water and different organic solvents. J Mol Liq 225:662-671

47. Khlyustova A, Sirotkin N, Evdokimova O, Prysiazhnyi V, Titov V (2018) Efficacy of underwater AC diaphragm discharge in generation of reactive species in aqueous solutions. J Electrostat 96:76-84

48. Vinodgopal K, Peller J (2003) Hydroxyl radical-mediated advanced oxidation processes for textile dyes: a comparison of the radiolytic and sonolytic degradation of the monoazo dye acid orange 7. Res Chem Intermed 29:307-316

49. Ying W, Weiwei W, Yingchao J, Hong L, Qiuhong W (2016) Evidence of hydroxyl radical created by DBD plasma in water and its application in dye wastewater remediation. Funct Mater 23:344-350

50. Hassaan MA, El Nemr A (2017) Advanced oxidation processes for textile wastewater treatment. Int J Photochem Photobiol $1: 27-35$

51. Buxton GV, Greenstock CL, Helman WP, Ross AB (1988) Critical review of rate constants for reactions of hydrated electrons, hydrogen atoms and hydroxyl radicals $\left(\mathrm{OH} / \mathrm{O}^{-}\right)$in aqueous solution. J Phys Chem Ref Data 17:513-886

52. Locke BR, Shih KY (2011) Review of the methods to form hydrogen peroxide in electrical discharge plasma with liquid water. Plasma Sources Sci Technol 20:034006

53. Byrappa K, Subramani AK, Ananda S, Rai KL, Dinesh R, Yoshimura $M$ (2006) Photocatalytic degradation of rhodamine B dye using hydrothermally synthesized ZnO. Bull Mater Sci 29:433-438

54. Mehrdad A, Hashemzadeh R (2009) Determination of activation energy for the degradation of rhodamine $B$ in the presence of hydrogen peroxide and some metal oxide. J Chem Soc Pak 31:738-743

55. Minakata D, Li K, Westerhoff P, Crittenden J (2009) Development of a group contribution method to predict aqueous phase hydroxyl radical ( $\mathrm{HO}$ ) reaction rate constants. Environ Sci Technol 43:6220-6227
56. Benetoli LODB, Cadorin BM, Postiglione CDS, Souza IGD, Debacher NA (2011) Effect of temperature on methylene blue decolorization in aqueous medium in electrical discharge plasma reactor. J Braz Chem Soc 22:1669-1678

57. McKay G, Dong MM, Kleinman JL, Mezyk SP, Rosario-Ortiz FL (2011) Temperature dependence of the reaction between the hydroxyl radical and organic matter. Environ Sci Technol 45:6932-6937

58. Ou XX, Wang C, Su Y, Zhang FJ, Yang GJ, Wang LY (2011) Degradation of rhodamine $B$ in aqueous solution by Fenton's reagent. Adv Mater Res 233-235:737-740

59. Ferrari C, Chen H, Lavezza R, Santinelli C, Longo I, Bramanti E (2013) Photodegradation of rhodamine B using the microwave/ UV/ $\mathrm{H}_{2} \mathrm{O}_{2}$ : effect of temperature. Int J Photoenergy 2013:854857

60. Ma J, Ma W, Song W, Chen C, Tang Y, Zhao J, Tang Y, Zang L (2006) Fenton degradation of organic pollutants in the presence of low-molecular-weight organic acids: cooperative effect of quinone and visible light. Environ Sci Technol 40:618-624

61. Buogo S, Plocek J, Vokurka K (2009) Efficiency of energy conversion in underwater spark discharges and associated bubble oscillations: experimental results. Acta Acust 95:46-59

62. Foster JE, Weatherford B, Gillman E, Yee B (2010) Underwater operation of a DBD plasma jet. Plasma Sources Sci Technol 19:025001

63. Izdebski T, Dors M, Mizeraczyk J (2014) Energy emissions of spark discharge under water. Eur Chem Bull 3:811-814

64. Merouani S, Hamdaoui O, Saoudi F, Chiha M (2010) Sonochemical degradation of rhodamine $B$ in aqueous phase: effects of additives. Chem Eng J 158:550-557

Publisher's Note Springer Nature remains neutral with regard to jurisdictional claims in published maps and institutional affiliations. 